Kragujevac Journal of Mathematics

Volume 42(2) (2018), Pages 259-273.

\title{
VECTOR VALUED HYPERSTRUCTURES
}

\author{
V. MIOVSKA ${ }^{1}$, V. CELAKOSKA-JORDANOVA ${ }^{1}$, AND B. DAVVAZ $^{2}$
}

\begin{abstract}
Vector valued hyperstructures, i.e., $(n, m)$-hyperstructures, where $n=$ $m+k, k \geq 1$, as a generalization of vector valued structures and $n$-ary hyperstructures are introduced and supported by many examples. We have presented some initial properties about $(n, m)$-hypersemigroups and $(n, m)$-hypergroups. Moreover, by properly defining regular and strongly regular binary relations, from vector valued hypersemigroups (hypergroups) we obtain "ordinary" vector valued semigroups (groups) on quotients.
\end{abstract}

\section{Introduction AND BAsic Definitions}

An $(n, m)$-groupoid is a nonempty set $G$ with one vector valued operation, i.e., an operation [ ] : $G^{n} \rightarrow G^{m}$, where $n \geq m$. Such a structure $(G,[])$ is called a vector valued groupoid as well. Vector valued groupoids were investigated in [15] and other special vector valued structures such as $(n, m)$-semigroups and $(n, m)$-groups were investigated in $[1,3,4,10-13,16]$. A good expository paper on vector valued structures is [2]. Compared with the papers devoted to $n$-ary structures, the number of the papers devoted to vector valued structures is smaller. Having in mind some recent works on $n$-ary hyperstructures such as [6-9], we define the notion of vector valued hypergroupoid and present some initial concepts, examples and results.

Let $H$ be a nonempty set and let $n, m$ be positive integers such that $n \geq m$. We denote by $\mathcal{P}^{*}(H)$ the set of all nonempty subsets of $H$ and by $H^{n}$ the $n$-th Cartesian product of $H, H \times \cdots \times H$, where $H$ appears $n$ times.

\footnotetext{
Key words and phrases. ( $n, m)$-hyperoperation, $(n, m)$-hypergroupoid, $(n, m)$-hypersemigroup, $(n, m)$-hyperquasigroup, $(n, m)$-hypergroup, regular relation, strongly regular relation.

2010 Mathematics Subject Classification. Primary: 20M10. Secondary: 20N99, 20 N20.

Received: December 25, 2016.

Accepted: March 06, 2017.
} 
Definition 1.1. Let [ ] be a mapping [ ] : $H^{n} \rightarrow\left(\mathcal{P}^{*}(H)\right)^{m}$ from the $n$-th Cartesian product of $H$ to the $m$-th Cartesian product of $\mathcal{P}^{*}(H)$. Then [ ] is called an $(n, m)$ hyperoperation on $H$ or, if it is not necessary to emphasize the integers $n$ and $m$, then we will say that [] is a vector valued hyperoperation instead of $(n, m)$-hyperoperation.

In this sense, a $(2,1)$-hyperoperation on $H$ means a binary hyperoperation on $H$ and an $(n, 1)$-hyperoperation means an $n$-ary hyperoperation on $H$.

Definition 1.2. A sequence of $m n$-ary hyperoperations [ ] $: H^{n} \rightarrow \mathcal{P}^{*}(H), s \in$ $\{1,2, \ldots, m\}$, can be associated to [ ] by putting

$$
\left[a_{1} \ldots a_{n}\right]_{s}=B_{s} \Leftrightarrow\left[a_{1} \ldots a_{n}\right]=\left(B_{1}, \ldots, B_{m}\right),
$$

for all $a_{1}, \ldots, a_{n} \in H$. Then, we call [ ] the $s$-th component hyperoperation of [ ] and write []$=\left([]_{1}, \ldots,[]_{m}\right)$.

Note that there is a unique $(n, m)$-hyperoperation on $H$ whose component hyperoperations are []$_{s}$.

An $(n, m)$-hyperoperation [ ] on $H$ is extended to subsets $A_{1}, A_{2}, \ldots, A_{n}$ of $H$ in a natural way, i.e.,

$$
\left[A_{1} A_{2} \ldots A_{n}\right]=\left(\left[A_{1} A_{2} \ldots A_{n}\right]_{1},\left[A_{1} A_{2} \ldots A_{n}\right]_{2}, \ldots,\left[A_{1} A_{2} \ldots A_{n}\right]_{m}\right),
$$

where $\left[A_{1} A_{2} \ldots A_{n}\right]_{s}=\cup\left\{\left[a_{1}^{n}\right]_{s} \mid a_{i} \in A_{i}, i=1,2, \ldots, n\right\}$ and $s=1,2, \ldots, m$.

Note that $C_{1}^{p} \subseteq B_{1}^{p}$ if and only if $C_{i} \subseteq B_{i}$, for $i=1, \ldots, p$, and, $x_{1}^{p} \in C_{1}^{p}$ if and only if $x_{i} \in C_{i}$ for $i=1, \ldots, p$.

Definition 1.3. An algebraic structure $\boldsymbol{H}=(H,[])$, where [ ] is an $(n, m)$-ary hyperoperation defined on a nonempty set $H$, is called an $(n, m)$-hypergroupoid or vector valued hypergroupoid. If []$=\left([]_{1}, \ldots,[]_{m}\right)$, we denote by $\left(H ;[]_{1}, \ldots,[]_{m}\right)$ the component hypergroupoid of $\boldsymbol{H}$ and $\left(H,[]_{j}\right)$ is the $j$-th component $n$-ary hypergroupoid of $\boldsymbol{H}$.

Identifying the set $\{x\}$ with the element $x$, any $(n, m)$-groupoid is an $(n, m)$ hypergroupoid.

Throughout the paper, we use the following simplified notation. The elements of $H^{n}$, i.e., the sequences $\left(x_{1}, x_{2}, \ldots, x_{n}\right)$ will be denoted by $x_{1} x_{2} \ldots x_{n}$ or $x_{1}^{n}$. The symbol $x_{i}^{j}$ will denote the sequence $x_{i} x_{i+1} \ldots x_{j}$ of elements of $H$ when $i \leq j$ and the empty symbol when $i>j$. If $x_{i+1}=x_{i+2}=\cdots=x_{i+r}=x$, then the sequence $x_{i+1}^{i+r}$ is denoted by $\stackrel{(r)}{x}$. Under this convention the sequence $x_{1} \ldots x_{i} \underbrace{x \ldots x}_{r} x_{i+r+1} \ldots x_{n}$ will be denoted by $x_{1}^{i} \stackrel{(r)}{x} x_{i+r+1}^{n}$.

In what follows we will assume that $n$ and $m$ are such that $n>m$, i.e., $n=m+k$, for $k \geq 1$.

Definition 1.4. An $(n, m)$-hyperoperation is said to be $(i, j)$-associative if for all $x_{1}, \ldots, x_{n+k} \in H$

$$
\left[x_{1}^{i}\left[x_{i+1}^{i+n}\right] x_{i+n+1}^{n+k}\right]=\left[x_{1}^{j}\left[x_{j+1}^{j+n}\right] x_{j+n+1}^{n+k}\right],
$$


and weakly $(i, j)$-associative if for all $x_{1}, \ldots, x_{n+k} \in H$

$$
\left[x_{1}^{i}\left[x_{i+1}^{i+n}\right] x_{i+n+1}^{n+k}\right]_{s} \cap\left[x_{1}^{j}\left[x_{j+1}^{j+n}\right] x_{j+n+1}^{n+k}\right]_{s} \neq \emptyset,
$$

holds for fixed $i$ and $j$, where $1 \leq i<j \leq n$ and for every $s \in\{1,2, \ldots, m\}$.

If the above conditions are satisfied for all $i, j \in\{1,2, \ldots, n\}$, then we say that the operation [] is associative (weakly associative, respectively). An ( $n, m)$-hypergroupoid with an associative operation (weakly associative operation) is called an $(n, m)$-hypersemigroup (weak $(n, m)$-hypersemigroup).

Definition 1.5. An $(n, m)$-hypergroupoid $(H,[])$ is partially $i$-cancellative if there exists a sequence $a_{1}^{k} \in H^{k}$ such that

$$
\left[a_{1}^{i} x_{1}^{m} a_{i+1}^{k}\right]=\left[a_{1}^{i} y_{1}^{m} a_{i+1}^{k}\right] \Rightarrow x_{1}^{m}=y_{1}^{m},
$$

for all $x_{1}^{m}, y_{1}^{m} \in H^{m}$ and some $i \in\{0,1, \ldots, k\}$. The sequence $a_{1}^{k}$ is called $i$-cancellable. If this implication holds for all $i=0,1, \ldots, k$, then we say that $(H,[])$ is partially cancellative and the sequence $a_{1}^{k}$ is cancellable. An $(n, m)$-hypergroupoid in which this implication holds for some $i \in\{0,1, \ldots, k\}$ and all sequences $a_{1}^{k} \in H^{k}$ is said to be $i$-cancellative. For $i=0(i=k)$ we say that $(H,[])$ is right cancellative (left cancellative). If $(H,[])$ is $i$-cancellative for every $i=0,1, \ldots, k$, then it is said to be cancellative. An $(n, m)$-hypergroupoid is strongly $i$-cancellative if for all $a_{1}^{k} \in H^{k}$ the following implication holds:

$$
\left[a_{1}^{i} X_{1}^{m} a_{i+1}^{k}\right]=\left[a_{1}^{i} Y_{1}^{m} a_{i+1}^{k}\right] \Rightarrow X_{1}^{m}=Y_{1}^{m},
$$

where $X_{i}, Y_{i} \subseteq H$ and some $i \in\{0,1, \ldots, k\}$. If this implication holds for all $i \in$ $\{0,1, \ldots, k\}$ we say that $(H,[])$ is strongly cancellative and the sequence $a_{1}^{k}$ is strongly cancellable. If there exists a sequence $a_{1}^{k} \in H^{k}$ such that the above implication holds, then we say that the $(n, m)$-hypergroupoid is partially strongly cancellative.

Remark 1.1. The definition of partially $i$-cancellative (partially cancellative) $(n, m)$ hypergroupoid for $m=1$ corresponds to the definition of weakly $i$-cancellative (weakly cancellative) $n$-ary hypergroupoid. Note that, if an $(n, m)$-hypergroupoid is strongly $i$-cancellative (strongly cancellative), then it is partially $i$-cancellative (partially cancellative).

Definition 1.6. Let $(H,[])$ be an $(n, m)$-hypergroupoid. A sequence $e_{1}^{k} \in H^{k}$ is called an $i$-neutral polyad if

$$
\left[x_{1}^{i} e_{1}^{k} x_{i+1}^{m}\right]=\left(\left\{x_{1}\right\},\left\{x_{2}\right\} \ldots,\left\{x_{m}\right\}\right) .
$$

We write this identity in the form $\left[x_{1}^{i} e_{1}^{k} x_{i+1}^{m}\right]=x_{1}^{m}$, for all $x_{1}^{m} \in H^{m}$. A 0-neutral polyad is also called left neutral and an $m$-neutral polyad is also called right neutral. A polyad that is $i$-neutral for each $i \in\{0,1, \ldots, m\}$ is called a neutral polyad.

If there exits $e \in H$ such that for any sequence $x_{1}^{m} \in H^{m}$ the relation

$$
x_{1}^{m} \in\left[x_{1}^{i} \stackrel{(k)}{e} x_{i+1}^{m}\right]
$$


holds for all $i=0, \ldots, m$, then we say that $e$ is a weak neutral element in $\boldsymbol{H}$. If

$$
\left[x_{1}^{i} \stackrel{(k)}{e} x_{i+1}^{m}\right]=\left(\left\{x_{1}\right\},\left\{x_{2}\right\} \ldots,\left\{x_{m}\right\}\right)
$$

holds for any $x_{1}^{m} \in H^{m}$ and fixed $i$, where $i \in\{0,1, \ldots, m\}$, then we say that $e$ is $i$ neutral element in $H$ and it is called a neutral element in $H$ if the equation (1.2) holds for every $i=0,1, \ldots, m$. We write the identity (1.2) in the form $\left[x_{1}^{i} \stackrel{(k)}{e} x_{i+1}^{m}\right]=x_{1}^{m}$.

Definition 1.7. An $(n, m)$-hypergroupoid $(H,[])$ is called an $(n, m)$-hyperquasigroup if for every $a_{1}^{n} \in H^{n}$ there exists $x_{1}^{m} \in H^{m}$ such that

$$
a_{k+1}^{n} \in\left[a_{1}^{i} x_{1}^{m} a_{i+1}^{k}\right] \text {, }
$$

for every $i=0,1, \ldots, k$.

Definition 1.8. An $(n, m)$-hyperquasigroup that is an (n,m)-hypersemigroup (weak ( $n, m)$-hypersemigroup) is called $(n, m)$-hypergroup (weak $(n, m)$-hypergroup).

Definition 1.9. An $(n, m)$-hypergroupoid $(H,[])$ is called $(i, j)$-commutative if the equality $\left[a_{1}^{n}\right]=\left[a_{1}^{i} a_{j} a_{i+2}^{j} a_{i} a_{j+2}^{n}\right]$ holds for fixed $i, j$ such that $0 \leq i<j \leq n-1$ and for every sequence $a_{1}^{n} \in H^{n}$. If this equation holds for every $i, j$ and for every sequence $a_{1}^{n} \in H^{n}$, then $(H,[])$ is called commutative (n,m)-hypergroupoid. In that case $\left[a_{1}^{n}\right]=\left[a_{\sigma(1)}^{\sigma(n)}\right]$, where $\sigma \in S_{n}$ and for every sequence $a_{1}^{n} \in H^{n}$. An $(n, m)$ hypergroupoid $(H,[])$ is called weakly commutative if $\bigcap_{\sigma \in S_{n}}\left[a_{\sigma(1)}^{\sigma(n)}\right]_{s} \neq \emptyset$ for every sequence $a_{1}^{n} \in H^{n}$ and every $s=1,2, \ldots, m$.

Definition 1.10. Let $(H,[])$ and $\left(H^{\prime},[]^{\prime}\right)$ be $(n, m)$-hypergroupoids. A mapping $\varphi: H \rightarrow H^{\prime}$ is:

a) a strong homomorphism if and only if $\varphi\left(\left[a_{1}^{n}\right]_{s}\right)=\left[\varphi\left(a_{1}\right) \ldots \varphi\left(a_{n}\right)\right]_{s}^{\prime}$;

b) an inclusion homomorphism if and only if $\varphi\left(\left[a_{1}^{n}\right]_{s}\right) \subseteq\left[\varphi\left(a_{1}\right) \ldots \varphi\left(a_{n}\right)\right]_{s}^{\prime}$;

c) a weak homomorphism if and only if $\varphi\left(\left[a_{1}^{n}\right]_{s}\right) \cap\left[\varphi\left(a_{1}\right) \ldots \varphi\left(a_{n}\right)\right]_{s}^{\prime} \neq \varnothing$, for every $s=1,2, \ldots, n$.

If $\varphi$ is a bijective mapping and a strong homomorphism, then it is called an isomorphism, and it is called an automorphism if $\varphi$ is defined on the same $(n, m)$ hypergroupoid.

\section{EXAMPLES}

Example 2.1. Let $H$ be the set $\mathbb{Z}$ of integers and let [ ] be defined as follows: $\left[x_{1}^{4}\right]=\left(\left\{x_{1}, x_{3}\right\},\left\{x_{2}, x_{4}\right\}\right)$. By a direct verification one can show that the component operations are:

$$
\begin{aligned}
{\left[\left[x_{1}^{4}\right] x_{5}^{6}\right]_{1} } & \left.=\left[\left\{x_{1}, x_{3}\right\}\left\{x_{2}, x_{4}\right\} x_{5} x_{6}\right\}\right]_{1} \\
& =\left[x_{1} x_{2} x_{5} x_{6}\right]_{1} \cup\left[x_{1} x_{4} x_{5} x_{6}\right]_{1} \cup\left[x_{3} x_{2} x_{5} x_{6}\right]_{1} \cup\left[x_{3} x_{4} x_{5} x_{6}\right]_{1} \\
& =\left\{x_{1}, x_{5}\right\} \cup\left\{x_{1}, x_{5}\right\} \cup\left\{x_{3}, x_{5}\right\} \cup\left\{x_{3}, x_{5}\right\}=\left\{x_{1}, x_{3}, x_{5}\right\}, \\
{\left[\left[x_{1}^{4}\right] x_{5}^{6}\right]_{2} } & \left.=\left[\left\{x_{1}, x_{3}\right\}\left\{x_{2}, x_{4}\right\} x_{5} x_{6}\right\}\right]_{2}
\end{aligned}
$$




$$
\begin{aligned}
& =\left[x_{1} x_{2} x_{5} x_{6}\right]_{2} \cup\left[x_{1} x_{4} x_{5} x_{6}\right]_{2} \cup\left[x_{3} x_{2} x_{5} x_{6}\right]_{2} \cup\left[x_{3} x_{4} x_{5} x_{6}\right]_{2} \\
& =\left\{x_{2}, x_{6}\right\} \cup\left\{x_{4}, x_{6}\right\} \cup\left\{x_{2}, x_{6}\right\} \cup\left\{x_{4}, x_{6}\right\}=\left\{x_{2}, x_{4}, x_{6}\right\}
\end{aligned}
$$

So,

$$
\begin{aligned}
{\left[\left[x_{1}^{4}\right] x_{5}^{6}\right] } & =\left(\left\{x_{1}, x_{3}, x_{5}\right\},\left\{x_{2}, x_{4}, x_{6}\right\}\right), \\
{\left[x_{1}\left[x_{2}^{5}\right] x_{6}\right]_{1} } & =\left[x_{1}\left\{x_{2}, x_{4}\right\}\left\{x_{3}, x_{5}\right\} x_{6}\right]_{1} \\
& =\left[x_{1} x_{2} x_{3} x_{6}\right]_{1} \cup\left[x_{1} x_{2} x_{5} x_{6}\right]_{1} \cup\left[x_{1} x_{4} x_{3} x_{6}\right]_{1} \cup\left[x_{1} x_{4} x_{5} x_{6}\right]_{1} \\
& =\left\{x_{1}, x_{3}\right\} \cup\left\{x_{1}, x_{5}\right\}=\left\{x_{1}, x_{3}, x_{5}\right\}, \\
{\left[x_{1}\left[x_{2}^{5}\right] x_{6}\right]_{2} } & =\left[x_{1}\left\{x_{2}, x_{4}\right\}\left\{x_{3}, x_{5}\right\} x_{6}\right]_{2} \\
& =\left[x_{1} x_{2} x_{3} x_{6}\right]_{2} \cup\left[x_{1} x_{2} x_{5} x_{6}\right]_{2} \cup\left[x_{1} x_{4} x_{3} x_{6}\right]_{2} \cup\left[x_{1} x_{4} x_{5} x_{6}\right]_{2} \\
& =\left\{x_{2}, x_{6}\right\} \cup\left\{x_{4}, x_{6}\right\}=\left\{x_{2}, x_{4}, x_{6}\right\} .
\end{aligned}
$$

So,

$$
\begin{aligned}
{\left[x_{1}\left[x_{2}^{5}\right] x_{6}\right] } & =\left(\left\{x_{1}, x_{3}, x_{5}\right\},\left\{x_{2}, x_{4}, x_{6}\right\}\right), \\
{\left[x_{1}^{2}\left[x_{3}^{6}\right]\right]_{1} } & =\left[x_{1} x_{2}\left\{x_{3}, x_{5}\right\}\left\{x_{4} x_{6}\right\}\right]_{1} \\
& =\left[x_{1} x_{2} x_{3} x_{4}\right]_{1} \cup\left[x_{1} x_{2} x_{3} x_{6}\right]_{1} \cup\left[x_{1} x_{2} x_{5} x_{4}\right]_{1} \cup\left[x_{1} x_{2} x_{5} x_{6}\right]_{1} \\
& =\left\{x_{1}, x_{3}\right\} \cup\left\{x_{1}, x_{3}\right\} \cup\left\{x_{1}, x_{5}\right\} \cup\left\{x_{1}, x_{5}\right\}=\left\{x_{1}, x_{3}, x_{5}\right\}, \\
{\left[x_{1}^{2}\left[x_{3}^{6}\right]\right]_{2} } & =\left[x_{1} x_{2}\left\{x_{3}, x_{5}\right\}\left\{x_{4} x_{6}\right\}\right]_{2} \\
& =\left[x_{1} x_{2} x_{3} x_{4}\right]_{2} \cup\left[x_{1} x_{2} x_{3} x_{6}\right]_{2} \cup\left[x_{1} x_{2} x_{5} x_{4}\right]_{2} \cup\left[x_{1} x_{2} x_{5} x_{6}\right]_{2} \\
& =\left\{x_{2}, x_{4}\right\} \cup\left\{x_{2}, x_{6}\right\} \cup\left\{x_{2}, x_{4}\right\} \cup\left\{x_{2}, x_{6}\right\}=\left\{x_{2}, x_{4}, x_{6}\right\} .
\end{aligned}
$$

So, $\left[x_{1}^{2}\left[x_{3}^{6}\right]\right]=\left(\left\{x_{1}, x_{3}, x_{5}\right\},\left\{x_{2}, x_{4}, x_{6}\right\}\right)$ and, obviously, $\left[\left[x_{1}^{4}\right] x_{5}^{6}\right]=\left[x_{1}\left[x_{2}^{5}\right] x_{6}\right]=\left[x_{1}^{2}\left[x_{3}^{6}\right]\right]$, i.e., $(H,[])$ is a $(4,2)$-hypersemigroup.

Remark 2.1. Note that the set $H$ with any of the component 4-ary hyperoperations does not have to be a 4-hypersemigroup.

Remark 2.2. If $\left(H,[]_{1}\right)$ and $\left(H,[]_{2}\right)$ are, for example, ternary hypersemigroups, then $(H,[])$, where []$=\left([]_{1},[]_{2}\right)$, does not necessarily have to be a $(3,2)$-hypersemigroup. For instance, let $H=\{a, b, c\}$ and []$_{1}$ be the ternary hyperoperation defined as in Example 2.4 in [9] and [ $]_{2}$ be the ternary hyperoperation defined as in Example 4 in [7]. Both $\left(H,[]_{1}\right)$ and $\left(H,[]_{2}\right)$ are ternary hypersemigroups as it is shown in [9] and [7]. However, $\left(H ;[]_{1},[]_{2}\right)$ is not a $(3,2)$-hypersemigroup, since $\left[[b a a]_{a}\right]_{1}=$ $\left[[b a a]_{1}[b a a]_{2} a\right]_{1}=[b b a]_{1}=\{a, c\} \neq[b[a a a]]_{1}=\left[b[a a a]_{1}[a a a]_{2}\right]_{1}=[b a a]_{1}=b$.

The next example presents a weak $(4,2)$-hypersemigroup that is not a $(4,2)$-hypersemigroup.

Example 2.2. Let $H$ be the set $\mathbb{Z}$ of integers, $(\mathbb{Z},+)$ be the additive group of integers and let [ ] be defined as follows:

$$
\left[x_{1}^{4}\right]=\left(\left\{x_{1}, x_{1}+x_{3}\right\},\left\{x_{2}, x_{2}+x_{4}\right\}\right) .
$$


By a direct verification one can show that the component operations are:

$$
\begin{aligned}
{\left[\left[x_{1}^{4}\right] x_{5}^{6}\right]_{1}=} & {\left.\left[\left\{x_{1}, x_{1}+x_{3}\right\}\left\{x_{2}, x_{2}+x_{4}\right\} x_{5} x_{6}\right\}\right]_{1} } \\
= & {\left[x_{1} x_{2} x_{5} x_{6}\right]_{1} \cup\left[x_{1}\left(x_{2}+x_{4}\right) x_{5} x_{6}\right]_{1} } \\
& \cup\left[\left(x_{1}+x_{3}\right) x_{2} x_{5} x_{6}\right]_{1} \cup\left[\left(x_{1}+x_{3}\right)\left(x_{2}+x_{4}\right) x_{5} x_{6}\right]_{1} \\
= & \left\{x_{1}, x_{1}+x_{5}\right\} \cup\left\{x_{1}+x_{3}, x_{1}+x_{3}+x_{5}\right\} \\
= & \left\{x_{1}, x_{1}+x_{3}, x_{1}+x_{5}, x_{1}+x_{3}+x_{5}\right\}, \\
{\left[\left[x_{1}^{4}\right] x_{5}^{6}\right]_{2}=} & {\left.\left[\left\{x_{1}, x_{1}+x_{3}\right\}\left\{x_{2}, x_{2}+x_{4}\right\} x_{5} x_{6}\right\}\right]_{2} } \\
= & {\left[x_{1} x_{2} x_{5} x_{6}\right]_{2} \cup\left[x_{1}\left(x_{2}+x_{4}\right) x_{5} x_{6}\right]_{2} } \\
& \cup\left[\left(x_{1}+x_{3}\right) x_{2} x_{5} x_{6}\right]_{2} \cup\left[\left(x_{1}+x_{3}\right)\left(x_{2}+x_{4}\right) x_{5} x_{6}\right]_{2} \\
= & \left\{x_{2}, x_{2}+x_{6}\right\} \cup\left\{x_{2}+x_{4}, x_{2}+x_{4}+x_{6}\right\} \\
= & \left\{x_{2}, x_{2}+x_{4}, x_{2}+x_{6}, x_{2}+x_{4}+x_{6}\right\} .
\end{aligned}
$$

From here we obtain that

$$
\left[\left[x_{1}^{4}\right] x_{5}^{6}\right]=\left(\left\{x_{1}, x_{1}+x_{3}, x_{1}+x_{5}, x_{1}+x_{3}+x_{5}\right\},\left\{x_{2}, x_{2}+x_{4}, x_{2}+x_{6}, x_{2}+x_{4}+x_{6}\right\}\right) .
$$

In a similar way as in the previous step, one can show that

$$
\left[x_{1}\left[x_{2}^{5}\right] x_{6}\right]=\left(\left\{x_{1}, x_{1}+x_{3}, x_{1}+x_{3}+x_{5}\right\},\left\{x_{2}, x_{2}+x_{4}, x_{2}+x_{6}, x_{2}+x_{4}+x_{6}\right\}\right)
$$

and that

$$
\left[x_{1}^{2}\left[x_{3}^{6}\right]=\left(\left\{x_{1}, x_{1}+x_{3}, x_{1}+x_{3}+x_{5}\right\},\left\{x_{2}, x_{2}+x_{4}, x_{2}+x_{4}+x_{6}\right\}\right) .\right.
$$

Note that $\left[\left[x_{1}^{4}\right] x_{5}^{6}\right] \neq\left[x_{1}\left[x_{2}^{5}\right] x_{6}\right]$ and therefore $(H,[])$ is not a $(4,2)$-hypersemigroup. However, for $i=1,2,\left[\left[x_{1}^{4}\right] x_{5}^{6}\right]_{i} \cap\left[x_{1}\left[x_{2}^{5}\right] x_{6}\right]_{i} \neq \emptyset,\left[\left[x_{1}^{4}\right] x_{5}^{6}\right]_{i} \cap\left[x_{1}^{2}\left[x_{3}^{6}\right]\right]_{i} \neq \emptyset$ and $\left[x_{1}\left[x_{2}^{5}\right] x_{6}\right]_{i} \cap$ $\left[x_{1}^{2}\left[x_{3}^{6}\right]\right]_{i} \neq \emptyset$. Thus $(H,[])$ is a weak $(4,2)$-hypersemigroup.

Example 2.3. Let $H=\mathbb{Z}_{3}$ and let [ ] be a $(3,2)$-hyperoperation on $H$ defined by $\left[x_{1}^{3}\right]=\left(\max \left\{x_{1}, x_{3}\right\}, x_{2}\right)$. Then $(H,[])$ is partially left cancellative, since there is an element $0 \in \mathbb{Z}_{3}$ such that $\left[0 x_{1}^{2}\right]=\left[0 y_{1}^{2}\right] \Rightarrow\left(\max \left\{0, x_{2}\right\}, x_{1}\right)=\left(\max \left\{0, y_{2}\right\}, y_{1}\right) \Rightarrow$ $x_{1}=y_{1}, x_{2}=y_{2}$. It can be shown in a similar way that $(H,[])$ is partially right cancellative as well.

Example 2.4. The $(4,2)$-hypersemigroup defined in the Example 2.1 is a cancellative $(4,2)$-hypergroupoid. Namely, let $a_{1}^{2} \in H^{2}$. Then

$$
\begin{aligned}
{\left[a_{1}^{2} x_{1}^{2}\right]=\left[a_{1}^{2} y_{1}^{2}\right] } & \Rightarrow\left(\left\{a_{1}, x_{1}\right\},\left\{a_{2}, x_{2}\right\}\right)=\left(\left\{a_{1}, y_{1}\right\},\left\{a_{2}, y_{2}\right\}\right) \\
& \Rightarrow\left\{a_{1}, x_{1}\right\}=\left\{a_{1}, y_{1}\right\},\left\{a_{2}, x_{2}\right\}=\left\{a_{2}, y_{2}\right\} \\
& \Rightarrow x_{1}=y_{1}, x_{2}=y_{2} \\
& \Rightarrow x_{1}^{2}=y_{1}^{2}, \\
{\left[x_{1} a_{1}^{2} x_{2}\right]=\left[y_{1} a_{1}^{2} y_{2}\right] } & \Rightarrow\left(\left\{x_{1}, a_{2}\right\},\left\{a_{1}, x_{2}\right\}\right)=\left(\left\{y_{1}, a_{2}\right\},\left\{a_{1}, y_{2}\right\}\right) \\
& \Rightarrow\left\{x_{1}, a_{2}\right\}=\left\{y_{1}, a_{2}\right\},\left\{a_{1}, x_{2}\right\}=\left\{a_{1}, y_{2}\right\} \\
& \Rightarrow x_{1}=y_{1}, x_{2}=y_{2}
\end{aligned}
$$




$$
\Rightarrow \quad x_{1}^{2}=y_{1}^{2} \text {. }
$$

In a similar way one can show that $\left[x_{1}^{2} a_{1}^{2}\right]=\left[y_{1}^{2} a_{1}^{2}\right] \Rightarrow x_{1}^{2}=y_{1}^{2}$.

Example 2.5. Let $H=\mathbb{Z},(\mathbb{Z},+)$, be the additive group of integers and [ ] be a $(4,2)$ hyperoperation defined by $\left[x_{1}^{4}\right]=\left(x_{1}+x_{3}, x_{2}+x_{4}\right)$. It is strongly cancellative. Namely, let $\left[x_{1} x_{2} A B\right]=\left[x_{1} x_{2} C D\right]$. Then, $\left(x_{1}+A, x_{2}+B\right)=\left(x_{1}+C, x_{2}+D\right)$ implies that $A=C, B=D$. If $\left[x_{1} A B x_{4}\right]=\left[x_{1} C D x_{4}\right]$, then $\left(x_{1}+B, A+x_{4}\right)=\left(x_{1}+D, C+x_{4}\right)$, and thus, $B=D, A=C$. If $\left[A B x_{3} x_{4}\right]=\left[C D x_{3} x_{4}\right]$, then $\left(A+x_{3}, B+x_{4}\right)=\left(C+x_{3}, D+x_{4}\right)$, and thus, $A=C, B=D$.

Note that if $(H,[])$ is strongly cancellative, then it is cancellative as well. The converse is not true. For instance, if $(H,[])$ is defined as in Example 2.4 then $(H,[])$ is cancellative but it is not strongly cancellative, since $[12\{1,2\}\{2,4\}]=[1224] \not$ $\{1,2\}=\{2\}$ and $\{2,4\}=\{4\}$.

Example 2.6. Let $H=\mathbb{Z}_{4},\left(\mathbb{Z}_{4},+\right)$, be the additive group of integers modulo 4 and [ ] be a $(4,2)$-hyperoperation on $H$ defined by

$$
\left[x_{1}^{4}\right]=\left(\left\{x_{1}+x_{3}, \max \left\{x_{1}, x_{3}\right\}\right\},\left\{x_{2}+x_{4}, \max \left\{x_{2}, x_{4}\right\}\right\}\right) .
$$

Since $\left[00 x_{3} x_{4}\right]=\left(\left\{x_{3}, x_{3}\right\},\left\{x_{4}, x_{4}\right\}\right)=\left(x_{3}, x_{4}\right),\left[x_{1} 00 x_{4}\right]=\left(\left\{x_{1}, x_{1}\right\},\left\{x_{4}, x_{4}\right\}\right)=$ $\left(x_{1}, x_{4}\right)$ and $\left[x_{1} x_{2} 00\right]=\left(\left\{x_{1}, x_{1}\right\},\left\{x_{2}, x_{2}\right\}\right)=\left(x_{1}, x_{2}\right)$, it follows that 0 is a neutral element in $\boldsymbol{H}$.

Example 2.7. Let $H=\mathbb{Z}$, where $(\mathbb{Z},+)$ is the additive group of integers, and [ ] be a $(4,2)$-hyperoperation on $H$ defined by

$$
\left[x_{1}^{4}\right]=\left(\left\{x_{1}+x_{3}, x_{3}\right\},\left\{x_{2}+x_{4}, x_{4}\right\}\right) .
$$

Since $\left[00 x_{3} x_{4}\right]=\left(x_{3}, x_{4}\right) \ni\left(x_{3}, x_{4}\right),\left[x_{1} 00 x_{4}\right]=\left(\left\{x_{1}, 0\right\}, x_{4}\right) \ni\left(x_{1}, x_{4}\right)$ and $\left[x_{1} x_{2} 00\right]=$ $\left(\left\{x_{1}, 0\right\},\left\{x_{2}, 0\right\}\right) \ni\left(x_{1}, x_{2}\right)$, it follows that 0 is a weak neutral element in $\boldsymbol{H}$.

Example 2.8. Let $H=\mathbb{Z}_{2}$ and [ ] be a (3,2)-hyperoperation on $H$ defined by $\left[x_{1}^{3}\right]=$ $\left(\left\{x_{1}, x_{2}\right\},\left\{x_{2}, x_{3}\right\}\right)$. By a direct verification for 8 sequences from elements of $H$, one can show that the relation (1.3) has a solution for every $(x, y) \in H^{2}$ and thus it is $(3,2)$ hyperquasigroup. Since it is a hypersemigroup as well (it can be easily verified that $\left.\left[\left[x_{1}^{3}\right] x_{4}\right]=\left(\left\{x_{1}, x_{2}, x_{3}\right\},\left\{x_{2}, x_{3}, x_{4}\right\}\right)=\left[x_{1}\left[x_{2}^{4}\right]\right]\right)$ it follows that $\boldsymbol{H}$ is a (3,2)-hypergroup.

Example 2.9. Let $H=\mathbb{Z}$ and [ ] is a $(4,2)$-hyperoperation on $H$ defined by $\left[x_{1}^{4}\right]=$ $\left(\left\{x_{1}, x_{3}\right\},\left\{x_{2}, x_{4}\right\}\right)$. Clearly, $(H,[])$ is $(0,2)$-commutative and $(1,3)$-commutative $(4,2)$-hypergroupoid that is not $(0,1)$-commutative.

Example 2.10. Let $H=\mathbb{Z}$, where $(\mathbb{Z},+, \cdot)$ is the ring of integers and [ ], be a $(3,2)$ hyperoperation on $H$ defined by

$$
\left[x_{1}^{3}\right]=\left(\left\{x_{1}+x_{2}+x_{3}, 0\right\},\left\{x_{1} x_{2} x_{3}, 1\right\}\right) .
$$

Clearly, $(H,[])$ is a commutative $(3,2)$-hypergroupoid. 
Example 2.11. Let $H=\mathbb{Z}$, where $(\mathbb{Z},+, \cdot)$ is the ring of integers, and [ ] be a $(3,2)$ hyperoperation on $H$ defined by $\left[x_{1}^{3}\right]=\left(\left\{x_{1}+x_{2}+x_{3}, x_{1}\right\},\left\{x_{1} x_{2} x_{3}, x_{2}\right\}\right)$. Then

$$
\begin{aligned}
& {\left[x_{1} x_{2} x_{3}\right]_{1}=\left\{x_{1}+x_{2}+x_{3}, x_{1}\right\}=\left[x_{1} x_{3} x_{2}\right]_{1},} \\
& {\left[x_{2} x_{1} x_{3}\right]_{1}=\left\{x_{1}+x_{2}+x_{3}, x_{2}\right\}=\left[x_{2} x_{3} x_{1}\right]_{1},} \\
& {\left[x_{3} x_{1} x_{2}\right]_{1}=\left\{x_{1}+x_{2}+x_{3}, x_{3}\right\}=\left[x_{3} x_{2} x_{1}\right]_{1},}
\end{aligned}
$$

and thus $\bigcap_{\sigma \in S_{n}}\left[x_{\sigma(1)}^{\sigma(3)}\right]_{1}=\bigcap_{j=1}^{3}\left\{x_{1}+x_{2}+x_{3}, x_{j}\right\}=\left\{x_{1}+x_{2}+x_{3}\right\} \neq \emptyset$.

In a similar way, we obtain that

$$
\begin{aligned}
& {\left[x_{1} x_{2} x_{3}\right]_{2}=\left\{x_{1} x_{2} x_{3}, x_{2}\right\}=\left[x_{3} x_{2} x_{1}\right]_{2},} \\
& {\left[x_{2} x_{1} x_{3}\right]_{2}=\left\{x_{1} x_{2} x_{3}, x_{1}\right\}=\left[x_{3} x_{1} x_{2}\right]_{2},} \\
& {\left[x_{1} x_{3} x_{2}\right]_{2}=\left\{x_{1} x_{2} x_{3}, x_{3}\right\}=\left[x_{2} x_{3} x_{1}\right]_{2},}
\end{aligned}
$$

and thus $\bigcap_{\sigma \in S_{n}}\left[x_{\sigma(1)}^{\sigma(3)}\right]_{2}=\bigcap_{j=1}^{3}\left\{x_{1} x_{2} x_{3}, x_{j}\right\}=\left\{x_{1} x_{2} x_{3}\right\} \neq \emptyset$.

Therefore, $\boldsymbol{H}$ is a weakly commutative $(3,2)$-hypergroupoid.

\section{Some Results on $(n, m)$-Hyperstructures}

Proposition 3.1. Let $(H,[])$ be an $(n, m)$-hypersemigroup. The following conditions are equivalent:

(i) $(H,[])$ is strongly cancellative;

(ii) $(H,[])$ is strongly left and strongly right cancellative;

(iii) $(H,[])$ is strongly $i$-cancellative for some $0<i<k$.

Proof. Implications (i) $\Rightarrow$ (ii) and (i) $\Rightarrow$ (iii) are obvious.

(ii) $\Rightarrow(\mathrm{i})$ Let $(H,[])$ be an $i$-cancellative hypersemigroup for $i=0$ and $i=k$. If

$$
\left[a_{1}^{j} x_{1}^{m} a_{j+1}^{k}\right]=\left[a_{1}^{j} y_{1}^{m} a_{j+1}^{k}\right]
$$

for $1 \leq j \leq k-1$, then for all $b_{1}^{k} \in H^{k}$ we have

$$
\left[b_{1}^{k-j}\left[a_{1}^{j} x_{1}^{m} a_{j+1}^{k}\right] b_{k-j+1}^{k}\right]=\left[b_{1}^{k-j}\left[a_{1}^{j} y_{1}^{m} a_{j+1}^{k}\right] b_{k-j+1}^{k}\right] .
$$

From the associativity it follows that

$$
\left[b_{1}^{k-j} a_{1}^{j}\left[x_{1}^{m} a_{j+1}^{k} b_{k-j+1}^{k}\right]\right]=\left[b_{1}^{k-j} a_{1}^{j}\left[y_{1}^{m} a_{j+1}^{k} b_{k-j+1}^{k}\right]\right] .
$$

From the strong cancellativity it follows that

$$
\left[x_{1}^{m} a_{j+1}^{k} b_{k-j+1}^{k}\right]=\left[y_{1}^{m} a_{j+1}^{k} b_{k-j+1}^{k}\right],
$$

which implies that $x_{1}^{m}=y_{1}^{m}$.

(iii) $\Rightarrow($ i) Let $(H,[])$ be strongly $i$-cancellative hypersemigroup for some $0<i<k$. First we will show that if $(H,[])$ is strongly $i$-cancellative, then $(H,[])$ is strongly $(i+1)$-cancellative hypersemigroup.

We have

$$
\left[a_{1}^{i+1} x_{1}^{m} a_{i+2}^{k}\right]=\left[a_{1}^{i+1} y_{1}^{m} a_{i+2}^{k}\right]
$$




$$
\begin{aligned}
& \Rightarrow\left[b_{1}^{i-1}\left[a_{1}^{i+1} x_{1}^{m} a_{i+2}^{k}\right] b_{i}^{k}\right]=\left[b_{1}^{i-1}\left[a_{1}^{i+1} y_{1}^{m} a_{i+2}^{k}\right] b_{i}^{k}\right] \\
& \Rightarrow\left[b_{1}^{i-1} a_{1}\left[a_{2}^{i+1} x_{1}^{m} a_{i+2}^{k} b_{i}\right] b_{i+}^{k}\right]=\left[b_{1}^{i-1} a_{1}\left[a_{2}^{i+1} y_{1}^{m} a_{i+2}^{k} b_{i}\right] b_{i+1}^{k}\right] \\
& \Rightarrow\left[a_{2}^{i+1} x_{1}^{m} a_{i+2}^{k} b_{i}\right]=\left[a_{2}^{i+1} y_{1}^{m} a_{i+2}^{k} b_{i}\right] .
\end{aligned}
$$

From the strong $i$-cancellativity it follows that $x_{1}^{m}=y_{1}^{m}$. Now, we will show that if $(H,[])$ is strongly $i$-cancellative, then $(H,[])$ is strongly $(i-1)$-cancellative hypersemigroup

$$
\begin{aligned}
& {\left[a_{1}^{i-1} x_{1}^{m} a_{i}^{k}\right]=\left[a_{1}^{i-1} y_{1}^{m} a_{i}^{k}\right] } \\
\Rightarrow & {\left[b_{1}^{i+1}\left[a_{1}^{i-1} x_{1}^{m} a_{i}^{k}\right] b_{i+2}^{k}\right]=\left[b_{1}^{i+1}\left[a_{1}^{i-1} y_{1}^{m} a_{i}^{k}\right] b_{i+2}^{k}\right] } \\
\Rightarrow & {\left[b_{1}^{i}\left[b_{i+1} a_{1}^{i-1} x_{1}^{m} a_{i}^{k-1}\right] a_{k} b_{i+2}^{k}\right]=\left[b_{1}^{i}\left[b_{i+1} a_{1}^{i-1} y_{1}^{m} a_{i}^{k-1}\right] a_{k} b_{i+2}^{k}\right] } \\
\Rightarrow & {\left[b_{i+1} a_{1}^{i-1} x_{1}^{m} a_{i}^{k-1}\right]=\left[b_{i+1} a_{1}^{i-1} y_{1}^{m} a_{i}^{k-1}\right] } \\
\Rightarrow & x_{1}^{m}=y_{1}^{m} .
\end{aligned}
$$

Hence, $(H,[])$ is strongly cancellative hypersemigroup.

Proposition 3.2. If for some $j$ such that $1 \leq j \leq i-1$ the (n, m)-hypergroupoid $(H,[])$ is $(i-1, i+j-1)$-associative and partially strongly $(i+j-1)$-cancellative, then it is $(i-j-1, i-1)$-associative.

Proof. Let $(H,[])$ be an $(n, m)$-hypergroupoid that is $(i-1, i+j-1)$-associative and partially strongly $(i+j-1)$-cancellative. Then it follows

$$
\begin{aligned}
& {\left[a_{1}^{i+j-1}\left[x_{1}^{i-j-1}\left[x_{i-j}^{n+i-j-1}\right] x_{n+i-j}^{n+k}\right] a_{i+j}^{k}\right] } \\
= & {\left[a_{1}^{i-1}\left[a_{i}^{i+j-1} x_{1}^{i-j-1}\left[x_{i-j}^{n+i-j-1}\right] x_{n+i-j}^{n+k-j}\right] x_{n+k-j+1}^{n+k} a_{i+j}^{k}\right] } \\
= & {\left[a_{1}^{i-1}\left[a_{i}^{i+j-1} x_{1}^{i-j-1} x_{i-j}^{i-1}\left[x_{i}^{n+i-1}\right] x_{n+i}^{n+k-j}\right] x_{n+k-j+1}^{n+k} a_{i+j}^{k}\right] } \\
= & {\left[a_{1}^{i-1} a_{i}^{i+j-1}\left[x_{1}^{i-1}\left[x_{i}^{n+i-1}\right] x_{n+i}^{n+k-j} x_{n+k-j+1}^{n+k}\right] a_{i+j}^{k}\right] } \\
= & {\left[a_{1}^{i+j-1}\left[x_{1}^{i-1}\left[x_{i}^{n+i-1}\right] x_{n+i}^{n+k}\right] a_{i+j}^{k}\right] . }
\end{aligned}
$$

Using the fact that $(H,[])$ is partially strongly $(i+j-1)$-cancellative we obtain that

$$
\left[x_{1}^{i-j-1}\left[x_{i-j}^{n+i-j-1}\right] x_{n+i-j}^{n+k}\right]=\left[x_{1}^{i-1}\left[x_{i}^{n+i-1}\right] x_{n+i}^{n+k}\right],
$$

i.e., $(i-j-1, i-1)$-associativity holds in $(H,[])$ for $1 \leq j \leq i-1$.

Proposition 3.3. If a partially strongly $(i-1)$-cancellative $(i \geq 1)(n, m)$-hypergroupoid $(H,[])$ is $(i-1, i+j-1)$-associative for some $j \geq 1$ and $2 j \leq k-i$, then it is $(i+j-1, i+2 j-1)$-associative.

Proof. Since $(H,[])$ is partially strongly $(i-1)$-cancellative, it follows that there is a sequence $a_{1}^{k} \in H^{k}$ such that $\left[a_{1}^{i-1} x_{1}^{m} a_{i}^{k}\right]=\left[a_{1}^{i-1} y_{1}^{m} a_{i}^{k}\right] \Rightarrow x_{1}^{m}=y_{1}^{m}$. Then, using the $(i-1, i+j-1)$-associativity for some $j \geq 1$ and $2 j \leq k-i$, and we obtain that

$$
\begin{aligned}
& {\left[a_{1}^{i-1}\left[x_{1}^{i+j-1}\left[x_{i+j}^{i+j+n+1}\right] x_{i+j+n}^{n+k}\right] a_{i}^{k}\right] } \\
= & {\left[a_{1}^{i-1}\left[x_{1}^{i+2 j-1}\left[x_{i+2 j}^{i+2 j+n-1}\right] x_{i+2 j+n}^{n+k}\right] a_{i}^{k}\right] }
\end{aligned}
$$




$$
\begin{aligned}
& =\left[a_{1}^{i-1}\left[x_{1}^{j}\left[x_{j+1}^{i+j-1}\left[x_{i+j}^{i+j+n-1}\right] x_{i+j+n}^{n+k}\right] a_{i}^{i+j-1}\right] a_{i+j}^{k}\right] \\
& =\left[a_{1}^{i-1} x_{1}^{j}\left[x_{j+1}^{i+2 j-1}\left[x_{i+2 j}^{i+2 j+n-1}\right] x_{i+2 j+n}^{n+k} a_{i}^{i+j-1}\right] a_{i+j}^{k}\right] \\
& =\left[a_{1}^{i-1}\left[x_{1}^{j} x_{j+1}^{i+2 j-1}\left[x_{i+2 j}^{i+2 j+n-1}\right] x_{i+2 j+n}^{n+k}\right] a_{i}^{k}\right] \\
& =\left[a_{1}^{i-1}\left[x_{1}^{i+2 j-1}\left[x_{i+2 j}^{i+2 j+n-1}\right] x_{i+2 j+n}^{n+k}\right] a_{i}^{k}\right] .
\end{aligned}
$$

The $(n, m)$-hypergroupoid $(H,[])$ is partially strongly $(i-1)$-cancellative and therefore

$$
\left[\left[x_{i+j}^{i+j+n+1}\right] x_{i+j+n}^{n+k}\right]=\left[x_{1}^{i+2 j-1}\left[x_{i+2 j}^{i+2 j+n-1}\right] x_{i+2 j+n}^{n+k}\right],
$$

i.e., $(H,[])$ is $(i+j-1, i+2 j-1)$-associative.

Proposition 3.4. Let $(H,[])$ be an $(n, m)$-hypersemigroup and let $e$ be a neutral element in $H$. Then the following conditions are equivalent:

(i) the sequence $\stackrel{(k)}{e}$ is strongly cancellable;

(ii) the sequence $\stackrel{(k)}{e}$ is strongly $i$-cancellable for $i=0$ and $i=k$;

(iii) The sequence $\stackrel{(k)}{e}$ is strongly $i$-cancellable for some $0<i<k$.

Proof. The proof is obvious from the proof of the Proposition 3.1.

Proposition 3.5. Let $(H,[])$ be an $(i, i+j)$-associative $(n, m)$-hypergroupoid for $1 \leq j \leq i$. If there exists a sequence $e_{1}^{k} \in H^{k}$ such that the equality $\left[e_{1}^{i+j} x_{1}^{m} e_{i+j+1}^{k}\right]=x_{1}^{m}$ holds for every $x_{1}^{m} \in H^{m}$, then $(H,[])$ is an $(i-j, i)$-associative $(n, m)$-hypergroupoid.

Proof. Using the equation given in the supposition we obtain that

$$
\left[x_{1}^{i-j}\left[x_{i-j+1}^{i-j+m+k}\right] x_{i-j+m+k+1}^{m+2 k}\right]=\left[e_{1}^{i+j}\left[x_{1}^{i-j}\left[x_{i-j+1}^{i-j+m+k}\right] x_{i-j+m+k+1}^{m+2 k}\right] e_{i+j+1}^{k}\right] .
$$

Applying the $(i, i+j)$-associativity twice and using the given equation in the last step, one obtains that

$$
\begin{aligned}
& {\left[e_{1}^{i+j}\left[x_{1}^{i-j}\left[x_{i-j+1}^{i-j+m+k}\right] x_{i-j+m+k+1}^{m+2 k}\right] e_{i+j+1}^{k}\right] } \\
= & {\left[e_{1}^{i}\left[x_{1}^{i-j}\left[x_{i-j+1}^{i-j+m+k}\right] x_{i-j+m+k+1}^{m+2 k-j}\right] x_{m+2 k-j+1}^{m+2 k} e_{i+j+1}^{k}\right] } \\
= & {\left[e_{1}^{i}\left[e_{i+1}^{j} x_{1}^{i}\left[x_{i+1}^{i+m+k}\right] x_{i+m+k+1}^{m+2 k-j}\right] x_{m+2 k-j+1}^{m+2 k} e_{i+j+1}^{k}\right] } \\
= & {\left[e_{1}^{i+j}\left[x_{1}^{i}\left[x_{i+1}^{i+m+k}\right] x_{i+m+k+1}^{m+2 k}\right] e_{i+j+1}^{k}\right] } \\
= & {\left[x_{1}^{i}\left[x_{i+1}^{i+m+k}\right] x_{i+m+k+1}^{m+2 k}\right] . }
\end{aligned}
$$

Thus, $(H,[])$ is an $(i-j, i)$-associative hypergroupoid for $1 \leq j \leq i$.

Proposition 3.6. Let $(H,[])$ be an $(i, i+j)$-associative $(n, m)$-hypergroupoid for $j \geq 1$. If there exists a sequence $e_{1}^{k} \in H^{k}$ such that the equality $\left[e_{1}^{i} x_{1}^{m} e_{i+1}^{k}\right]=x_{1}^{m}$ holds for every $x_{1}^{m} \in H^{m}$ and if $i+2 j \leq k$, then $(H,[])$ is an $(i+j, i+2 j)$-associative $(n, m)$-hypergroupoid.

Proof. Using the equation given in the supposition we obtain that

$$
\left[x_{1}^{i+j}\left[x_{i+j+1}^{i+j+m+k}\right] x_{i+j+m+k+1}^{m+2 k}\right]=\left[e_{1}^{i}\left[x_{1}^{i+j}\left[x_{i+j+1}^{i+j+m+k}\right] x_{i+j+m+k+1}^{m+2 k}\right] e_{i+1}^{k}\right] .
$$


Applying the $(i, i+j)$-associativity three times and using the given equation in the last step, one obtains that

$$
\begin{aligned}
& {\left[e_{1}^{i}\left[x_{1}^{i+j}\left[x_{i+j+1}^{i+j+m+k}\right] x_{i+j+m+k+1}^{m+2 k}\right] e_{i+1}^{k}\right] } \\
= & {\left[e_{1}^{i} x_{1}^{j}\left[x_{j+1}^{i+j}\left[x_{i+j+1}^{i+j+m+k}\right] x_{i+j+m+k+1}^{m+2 k} e_{i+1}^{i+j}\right] e_{i+j+1}^{k}\right] } \\
= & {\left[e_{1}^{i} x_{1}^{j}\left[x_{j+1}^{i+2 j}\left[x_{i+2 j+1}^{i+2 j+m+k}\right] x_{i+2 j+m+k+1}^{m+2 k} e_{i+1}^{i+j}\right] e_{i+j+1}^{k}\right] } \\
= & {\left[e_{1}^{i}\left[x_{1}^{i+2 j}\left[x_{i+2 j+1}^{i+2 j+m+k}\right] x_{i+2 j+m+k+1}^{m+2 k}\right] e_{i+1}^{k}\right] } \\
= & {\left[x_{1}^{i+2 j}\left[x_{i+2 j+1}^{i+2 j+m+k}\right] x_{i+2 j+m+k+1}^{m+2 k}\right] . }
\end{aligned}
$$

Hence, $(H,[])$ is an $(i+j, i+2 j)$-associative hypergroupoid for $i+2 j \leq k$ and $j \geq 1$.

Proposition 3.7. Let $(H,[])$ be an $(n, m)$-hypersemigroup with an m-neutral element $e$ that satisfies the identity $\left[e x_{1}^{m} \stackrel{(k-1)}{e}\right]=x_{1}^{m}$. Then

a) $\left[\stackrel{(i)}{e} x_{1}^{m} \stackrel{(k-i)}{e}\right]=x_{1}^{m}$ for $2 \leq i \leq k$;

b) e is a neutral element.

Proof. a) First we will prove that $\left[\stackrel{(2)}{e} x_{1}^{m} \stackrel{(k-2)}{e}\right]=x_{1}^{m}$. Namely:

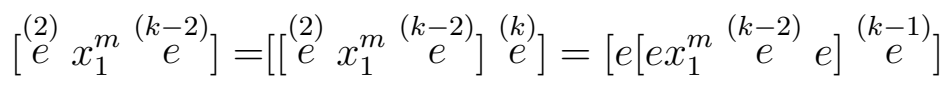

$$
\begin{aligned}
& =\left[e\left[e x_{1}^{m} \stackrel{(k-1)}{e}\right] \stackrel{(k-1)}{e}\right]=\left[e x_{1}^{m} \stackrel{(k-1)}{e}\right]=x_{1}^{m} .
\end{aligned}
$$

Iterating this procedure for every $3 \leq i \leq k$, using the condition and every result obtained in the previous step, we obtain that

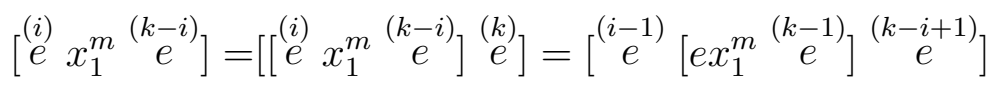

$$
\begin{aligned}
& =\left[\stackrel{(i-1)}{e} x_{1}^{m} \stackrel{(k-i+1)}{e}\right]=x_{1}^{m} \text {. }
\end{aligned}
$$

b) The proof follows from the supposition and a suitable application of a). Namely, $\left[x_{1} \stackrel{(k)}{e} x_{2}^{m}\right]=\left[\left[\begin{array}{lll}x_{1} & \stackrel{(k)}{e} & x_{2}^{m}\end{array}\right] \stackrel{(k)}{e}\right]=\left[x_{1}\left[\begin{array}{ll}\stackrel{(k)}{e} & x_{2}^{m} e\end{array}\right] \stackrel{(k-1)}{e}\right]=\left[x_{1} x_{2}^{m} e \stackrel{(k-1)}{e}\right]=\left[x_{1}^{m} \stackrel{(k)}{e}\right]=x_{1}^{m}$.

The next proposition is symmetrical to Proposition 3.7.

Proposition 3.8. Let $(H,[])$ be an $(n, m)$-hypersemigroup with a 0-neutral element $e$ that satisfies the identity $\left[{ }^{(k-1)} x_{1}^{m} e\right]=x_{1}^{m}$. Then

a) $\left[\stackrel{(i)}{e} x_{1}^{m} \stackrel{(k-i)}{e}\right]=x_{1}^{m}$ for $2 \leq i \leq k$;

b) e is a neutral element.

Proposition 3.9. If $(H,[])$ is an $(m+k, m)$-hypergroupoid such that $k<m$ and $(H,[])$ has a neutral element e, then $|H|=1$. 
Proof. Let $x \in H$ be arbitrarily chosen element. Then, $[\stackrel{(k)(m-k)(k)}{e} \stackrel{e}{e}]=\stackrel{(m-k)(k)}{e}$ and $[\stackrel{(k)(m-k)(k)}{e} \stackrel{e}{e}]=\stackrel{(k)(m-k)}{x}$, which implies that $x=e$, i.e., $|H|=1$.

Theorem 3.1. An $(n, m)$-hypersemigroup $(H,[])$ is an $(n, m)$-hypergroup if and only if the relation (1.3) holds for

a) $i=0$ and $i=k$ (i.e., $a_{k+1}^{n} \in\left[x_{1}^{m} a_{1}^{k}\right]$ and $a_{k+1}^{n} \in\left[a_{1}^{k} x_{1}^{m}\right]$, for all $\left.a_{1}^{n} \in H^{n}\right)$;

b) some $i, 1 \leq i<k-1$.

Proof. The direct statements are obvious.

a) $\Rightarrow(1.3)$ For the converse, let the relation (1.3) holds for $i=0$ and $i=k$. Then for $a_{1}^{k} \in H^{k}$ and $b_{1}^{m} \in H^{m}$, there is an $x_{1}^{m} \in H^{m}$ such that $b_{1}^{m} \in\left[x_{1}^{m} a_{1}^{k}\right]$ and for $a_{1}^{k} \in H^{k}$ and $x_{1}^{m} \in H^{m}$, there is an $y_{1}^{m}$ such that $x_{1}^{m} \in\left[a_{1}^{k} y_{1}^{m}\right]$. From here we obtain that

$$
b_{1}^{m} \in\left[\left[a_{1}^{k} y_{1}^{m}\right] a_{1}^{k}\right]=\left[a_{1}^{i}\left[a_{i+1}^{k} y_{1}^{m} a_{1}^{i}\right] a_{i+1}^{k}\right],
$$

for $1 \leq i \leq k-1$, so the relation (1.3) holds for every $i$, i.e., $(H,[])$ is an $(n, m)$ hypergroup.

$\mathrm{b}) \Rightarrow(1.3)$ Let the relation (1.3) holds for some $i, 1 \leq i<k-1$. Then, for $a_{1}^{k} \in H^{k}$ and $b_{1}^{m} \in H^{m}$, there is $x_{1}^{m} \in H^{m}$ such that $b_{1}^{m} \in\left[a_{1}^{i} x_{1}^{m} a_{i+1}^{k}\right]$. For $a_{i+1}^{(k)} \in H^{k}$ and

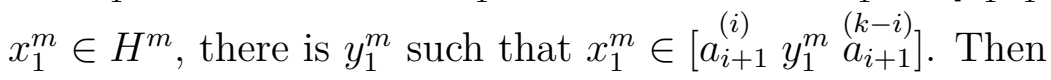

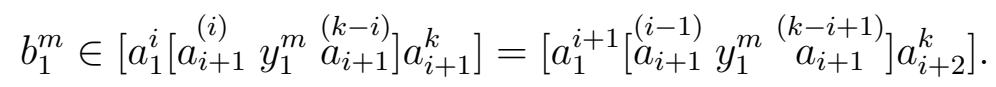

So, the relation (1.3) is solvable in the $i+1$ place, for all $1 \leq i \leq k-1$ and consequently, it is solvable in the $k$ place.

For $\stackrel{(k)}{a_{i}} \in H^{k}$ and $x_{1}^{m} \in H^{m}$, there is $z_{1}^{m} \in H^{m}$ such that $x_{1}^{m} \in\left[\stackrel{(i)}{a_{i}} z_{1}^{m} \stackrel{(k-i)}{a_{i}}\right]$. Then

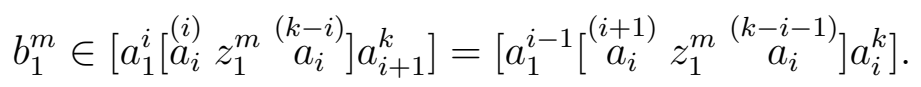

So, the relation (1.3) is solvable in the $i-1$ place, for all $1 \leq i \leq k-1$ and consequently, it is solvable in the 0 place.

Hence, $(H,[])$ is an $(n, m)$-hypergroup.

\section{Relations on $(n, m)$-HyPERstruCtURES}

Let $(H,[])$ be an $(n, m)$-hypersemigroup. An equivalence relation $\rho$ on $H$ is said to be

(a) regular if $a_{j} \rho b_{j}, j \in\{1,2, \ldots, n\}$ and $x \in\left[a_{1}^{n}\right]_{s}, s \in\{1,2, \ldots, m\}$, implies that there exists $y \in\left[b_{1}^{n}\right]_{s}, s \in\{1,2, \ldots, m\}$, such that $x \rho y$;

(b) strongly regular if $a_{j} \rho b_{j}, j \in\{1,2, \ldots, n\}$ implies that $x \rho y$, for every $x \in\left[a_{1}^{n}\right]_{s}$, $y \in\left[b_{1}^{n}\right]_{s}$ and $s \in\{1,2 \ldots, m\}$.

Theorem 4.1. Let $(H,[])$ be an $(n, m)$-hypersemigroup and $\rho$ be an equivalence relation on $H$. 
(i) If $\rho$ is regular, then $\left(H / \rho,[]^{\rho}\right)$ is a $(n, m)$-hypersemigroup, where the operation []$^{\rho}$ consists of $m$ component hyperoperations, []$^{\rho}=\left([]_{1}^{\rho}, \ldots,[]_{m}^{\rho}\right)$, each of which is defined as follows:

$$
\left[\rho\left(a_{1}\right) \rho\left(a_{2}\right) \ldots \rho\left(a_{n}\right)\right]_{s}^{\rho}=\left\{\rho(x) \mid x \in\left[a_{1}^{n}\right]_{s}\right\},
$$

for every $s \in\{1,2, \ldots, m\}$.

(ii) If $\left(H / \rho,[]^{\rho}\right)$ is a $(n, m)$-hypersemigroup, then $\rho$ is a regular relation.

(iii) The canonical projection $\pi: H \rightarrow H / \rho$ such that $\pi(a)=\rho(a)$ is a strong homomorphism on the $(n, m)$-hypersemigroups $(H,[])$ and $\left(H / \rho,[]^{\rho}\right)$.

(iv) If $(H,[])$ is a $(n, m)$-hypergroup, then $\left(H / \rho,[]^{\rho}\right)$ is a $(n, m)$-hypergroup as well.

Proof. (i) First, we will show that [ $]^{\rho}$ is a well defined operation on $H / \rho$. Let $\rho\left(a_{i}\right)=\rho\left(c_{i}\right)$, for $i=1,2, \ldots, n$. Clearly, $a_{i} \rho c_{i}$, for $i=1,2, \ldots, n$. Let $\rho(x) \in$ $\left[\rho\left(a_{1}\right) \rho\left(a_{2}\right) \ldots \rho\left(a_{n}\right)\right]_{s}^{\rho}$. Then $x \in\left[a_{1}^{n}\right]_{s}$. By the assumption, $\rho$ is a regular relation and thus, for every $x \in\left[a_{1}^{n}\right]_{s}$ there exists $y \in\left[c_{1}^{n}\right]_{s}, s=1,2, \ldots, m$, such that $x \rho y$. Since $\rho(x)=\rho(y)$ and $\rho(x)=\rho(y) \in\left[\rho\left(c_{1}\right) \rho\left(c_{2}\right) \ldots \rho\left(a_{n}\right)\right]_{s}^{\rho}$ it follows that $\left[\rho\left(a_{1}\right) \rho\left(a_{2}\right) \ldots \rho\left(a_{n}\right)\right]_{s}^{\rho} \subseteq\left[\rho\left(c_{1}\right) \rho\left(c_{2}\right) \ldots \rho\left(c_{n}\right)\right]_{s}^{\rho}, s=1,2, \ldots, m$. The converse inclusion can be shown in a similar way.

In order to prove the $(m+k, m)$-associativity, suppose that $\rho\left(a_{i}\right) \in H / \rho$, where $i=1,2, \ldots, m+2 k$ and let $\rho(z)$ belongs in

$$
\left[\left[\rho\left(a_{1}\right) \ldots \rho\left(a_{m+k}\right)\right]_{1}^{\rho} \ldots\left[\rho\left(a_{1}\right) \ldots \rho\left(a_{m+k}\right)\right]_{m}^{\rho} \rho\left(a_{m+k+1}\right) \ldots \rho\left(a_{m+2 k}\right)\right]_{s}^{\rho}
$$

for $s=1,2 \ldots, m$. Then, there exists $\rho\left(u_{\lambda}\right) \in\left[\rho\left(a_{1}\right) \rho\left(a_{2}\right) \ldots \rho\left(a_{m+k}\right)\right]_{\lambda}^{\rho}$, for $\lambda=$ $1,2, \ldots, m$ and $z \in\left[u_{1}^{m} a_{m+k+1}^{m+2 k}\right]_{s}$, for $s=1,2, \ldots, m$.

Clearly, $z \in\left[\left[a_{1}^{m+k}\right]_{1} \ldots,\left[a_{1}^{m+k}\right]_{m} a_{m+k+1}^{m+2 k}\right]_{s}=\left[a_{1}^{j}\left[a_{j+1}^{j+m+k}\right]_{1} \ldots\left[a_{j+1}^{j+m+k}\right]_{m} a_{j+m+k+1}^{m+2 k}\right]_{s}$. There exists $x_{\lambda} \in\left[a_{j+1}^{j+m+k}\right]_{\lambda}$, for $\lambda=1, \ldots, m$ and $j=1, \ldots, k$, such that $z \in$ $\left[a_{1}^{j} x_{1}^{m} a_{j+m+k+1}^{m+2 k}\right]_{s}$, for $s=1,2, \ldots, m$. Therefore,

$$
\begin{aligned}
\rho(z) \in & {\left[\rho\left(a_{1}\right) \ldots \rho\left(a_{j}\right)\left[\rho\left(a_{j+1}\right) \ldots \rho\left(a_{j+m+k}\right)\right]_{1}^{\rho} \ldots\left[\rho\left(a_{j+1}\right) \ldots \rho\left(a_{j+m+k}\right)\right]_{m}^{\rho}\right.} \\
& \left.\rho\left(a_{j+m+k+1}\right) \ldots \rho\left(a_{m+2 k}\right)\right]_{s}^{\rho}
\end{aligned}
$$

and thus

$$
\begin{aligned}
& {\left[\left[\rho\left(a_{1}\right) \ldots \rho\left(a_{m+k}\right)\right]_{1}^{\rho} \ldots\left[\rho\left(a_{1}\right) \ldots \rho\left(a_{m+k}\right)\right]_{m}^{\rho} \rho\left(a_{m+k+1}\right) \ldots \rho\left(a_{m+2 k}\right)\right]_{s}^{\rho} } \\
\subseteq & {\left[\rho\left(a_{1}\right) \ldots \rho\left(a_{j}\right)\left[\rho\left(a_{j+1}\right) \ldots \rho\left(a_{j+m+k}\right)\right]_{1}^{\rho} \ldots\left[\rho\left(a_{j+1}\right) \ldots \rho\left(a_{j+m+k}\right)\right]_{m}^{\rho}\right.} \\
& \left.\rho\left(a_{j+m+k+1}\right) \ldots \rho\left(a_{m+2 k}\right)\right]_{s}^{\rho}
\end{aligned}
$$

for $s=1,2, \ldots, m$ and $j=1,2, \ldots, k$.

The converse inclusion can be shown similarly.

(ii) Let $a_{i} \rho c_{i}$, for $i=1,2, \ldots, n$. Then, $\rho\left(a_{i}\right)=\rho\left(c_{i}\right), i=1,2, \ldots, n$ and so $\left[\rho\left(a_{1}\right) \rho\left(a_{2}\right) \ldots \rho\left(a_{n}\right)\right]_{s}^{\rho}=\left[\rho\left(c_{1}\right) \rho\left(c_{2}\right) \ldots \rho\left(c_{n}\right)\right]_{s}^{\rho}$, for $s=1,2, \ldots, m$. For every $x \in\left[a_{1}^{n}\right]_{s}$, $s=1,2, \ldots, m$, we have that $\rho(x) \in\left[\rho\left(c_{1}\right) \rho\left(c_{2}\right) \ldots \rho\left(c_{n}\right)\right]_{s}^{\rho}$, so there exists $y \in\left[c_{1}^{n}\right]_{s}$, such that $\rho(x)=\rho(y)$, and therefore $x \rho y$. 
(iii) We claim that $\pi\left(\left[a_{1}^{n}\right]_{s}\right)=\left[\pi\left(a_{1}\right) \pi\left(a_{2}\right) \ldots \pi\left(a_{n}\right)\right]_{s}^{\rho}, s=1,2, \ldots, m$. Let $\rho(a) \in$ $\pi\left(\left[a_{1}^{n}\right]_{s}\right), s=1,2, \ldots, m$. Then, there exists $a^{\prime} \in\left[a_{1}^{n}\right]_{s}$, such that $\rho(a)=\pi\left(a^{\prime}\right)$. So, $\rho(a)=\rho\left(a^{\prime}\right) \in\left[\rho\left(a_{1}\right) \rho\left(a_{2}\right) \ldots \rho\left(a_{n}\right)\right]_{s}^{\rho}=\left[\pi\left(a_{1}\right) \pi\left(a_{2}\right) \ldots \pi\left(a_{n}\right)\right]_{s}^{\rho}$. Thus, $\pi\left(\left[a_{1}^{n}\right]_{s}\right) \subseteq$ $\left[\pi\left(a_{1}\right) \pi\left(a_{2}\right) \ldots \pi\left(a_{n}\right)\right]_{s}^{\rho}, s=1,2, \ldots, m$.

For the converse inclusion, let $\rho(a) \in\left[\pi\left(a_{1}\right) \pi\left(a_{2}\right) \ldots \pi\left(a_{n}\right)\right]_{s}^{\rho}, s=1,2, \ldots, m$. Since $\pi\left(a_{i}\right)=\rho\left(a_{i}\right), i=1,2, \ldots, n$, it follows that $\rho(a) \in\left[\rho\left(a_{1}\right) \rho\left(a_{2}\right) \ldots \rho\left(a_{n}\right)\right]_{s}^{\rho}, s=$ $1,2, \ldots, m$. Then, there exists $a^{\prime} \in \rho(a)$ such that $a^{\prime} \in\left[a_{1}^{n}\right]_{s}, s=1,2, \ldots, m$. Thus, $\rho(a)=\rho\left(a^{\prime}\right)=\pi\left(a^{\prime}\right) \in \pi\left(\left[a_{1}^{n}\right]_{s}\right)$, so $\left[\pi\left(a_{1}\right) \pi\left(a_{2}\right) \ldots \pi\left(a_{n}\right)\right]_{s}^{\rho} \subseteq \pi\left(\left[a_{1}^{n}\right]_{s}\right), s=1,2, \ldots, m$.

(iv) Suppose that $(H,[])$ is a $(n, m)$-hypergroup, i.e., $(H,[])$ is a $(n, m)$-hypersemigroup such that for every $a_{1}^{n} \in H^{n}$, there is $x_{1}^{m} \in H^{m}$ such that $a_{k+1}^{m+k} \in\left[a_{1}^{j} x_{1}^{m} a_{j+1}^{k}\right]$, for every $j=0,1, \ldots, k$. Let $\rho\left(a_{i}\right) \in H / \rho, i=1,2, \ldots, n$. Then, there exists $t_{1}, t_{2}, \ldots, t_{m} \in H$ such that $a_{k+1}^{m+k} \in\left[a_{1}^{j} t_{1}^{m} a_{j+1}^{k}\right]$, for every $j=0,1, \ldots, k$. Thus, $\rho\left(a_{k+s}\right) \in\left[\rho\left(a_{1}\right) \ldots \rho\left(a_{j}\right) \rho\left(t_{1}\right) \ldots \rho\left(t_{m}\right) \rho\left(a_{j+1}\right) \ldots \rho\left(a_{k}\right)\right]_{s}^{\rho}$, for every $j=0,1, \ldots, k$ and $s=1,2, \ldots, m$. Therefore, $\left(H / \rho,[]^{\rho}\right)$ is a $(n, m)$-hypergroup.

Theorem 4.2. Let $(H,[])$ be an $(n, m)$-hypersemigroup and $\rho$ be a strongly regular relation on $H$. Then

(i) $\left(H / \rho,[]^{\rho}\right)$ is an $(n, m)$-semigroup;

(ii) if $(H,[])$ is an $(n, m)$-hypergroup, then $\left(H / \rho,[]^{\rho}\right)$ is an $(n, m)$-group.

Proof. (i) Let $\rho(x), \rho(y) \in\left[\rho\left(a_{1}\right) \rho\left(a_{2}\right) \ldots \rho\left(a_{n}\right)\right]_{s}^{\rho}, s=1,2, \ldots, m$. Then, $x, y \in$ $\left[a_{1}^{n}\right]_{s}$. Since $\rho$ is strongly regular, it follows that $x \rho y$, i.e., $\rho(x)=\rho(y)$. Thus, $\left|\left[\rho\left(a_{1}\right) \rho\left(a_{2}\right) \ldots \rho\left(a_{n}\right)\right]_{s}^{\rho}\right|=1$, for $s=1,2, \ldots, m$. Therefore, $\left(H / \rho,[]^{\rho}\right)$ is an $(n, m)$ semigroup.

(ii) It follows from (i) and Theorem 4.1(iv).

\section{Future Work}

We have started the investigation on vector valued hypersemigroups and vector valued hypergroups. As a future work we are planing to introduce a relation $\beta$ on a vector valued hypersemigroup (hypergroup) and to investigate the fundamental equivalence relation $\beta^{*}$ as the smallest equivalence relation on a vector valued hypersemigroup (hypergroup), such that $H / \beta^{*}$ would be a vector valued semigroup (group).

\section{REFERENCES}

[1] Ǵ. Čupona, Vector valued semigroups, Semigroup Forum 26 (1983), 65-74.

[2] Ǵ. Čupona, N. Celakoski, S. Markovski and D. Dimovki, Vector valued groupoids, semigroups and groups, Macedonian Academy of Sciences and Arts, Skopje, 1988, 1-78.

[3] Ǵ. Čupona and D. Dimovski, On a class of vector valued groups, Proceedings of the Conference "Algebra and Logic", Zagreb 1984; Novi Sad Institute of Mathematics, 1985, 29-37.

[4] Ǵ. Čupona, D. Dimovski and A. Samardžiski, Fully commutative vector valued groups, Prilozi MANU-Oddel. za mat.-teh. nauki, Skopje, VIII 2 (1987), 5-17.

[5] B. Davvaz, Polygroup Theory and Related Systems, World Scientific Publishing Co. 2013.

[6] B. Davvaz, W. A. Dudek and S. Mirvakili, Neutral Elements, Fundamental relations and n-ary hypersemigroups, Internat. J. Algebra Comput. Appl. 19 (2009), 567-583. 
[7] B. Davvaz, W. A. Dudek and T. Vougiouklis, A Generalization of n-ary Algebraic Systems, Comm. Algebra 37 (2009), 1248-1263.

[8] B. Davvaz and V. Leoreanu-Fotea, Ternary hypergroups (a survey), International Journal of Algebraic Hyperstructures and its Applications 1 (2014), 29-44.

[9] B. Davvaz and T. Vougiouklis, N-ary hypergroups, Iran. J. Sci. Technol. Trans. A Sci. 30 (2006), $165-174$.

[10] D. Dimovski, Some existence conditions for vector valued groups, God. Zb. Inst. Mat. Prir.-Mat. Fak. Univ. Kiril Metodij Skopje 33-34 (1982-83), 99-103.

[11] D. Dimovski, On $(m+k, m)$-groups for $k<m$, General Algebra and Discrete Mathematics, Denecke, K., Lüders, O., Eds., Heldermann-Verlag, Berlin, 1995, 93-99.

[12] B. Janeva, V. Miovska and V. Celakoska-Jordanova, On a class of n-groupoids, Proceedings of the II Congress of Mathematicans and Informaticians of Macedonia, Ohrid 2000, 2003, 25-30.

[13] S. Markovski, Free ( $V$; n, m)-groupoids, Mat. Bilten 16 (XLII) (1992), 5-16.

[14] F. Marty, Sur une Generalization de la Notion de Groupe, 8th Congress Math., Scandenaves, Stocholm, 1934, 45-49.

[15] B. Trpenovski and Ǵ. Čupona, $[m, n]$-groupoids, Bull. Soc. Math. Phys. Macédoine 21 (1970), 19-29 (in Macedonian).

[16] J. Ušan, (n,m)-groups in the light of the neutral operations, Math. Morav. 10 (2006), $107-147$.

${ }^{1}$ Institute of Mathematics,

Faculty of Natural Sciences and Mathematics,

Ss. Cyril and Methodius University,

Arhimedova 3, 1000 Skopje, R. Macedonia

Email address: miovska@pmf .ukim.mk

Email address: vesnacj@pmf.ukim.mk, celakoska@gmail.com

${ }^{2}$ Department of MAThematics,

YAZD UNIVERSITY,

YAZD, IRAN

Email address: davvaz@yazd.ac.ir 